\title{
Homogeneously polyanalytic kernels on the unit ball and the Siegel domain
}

\author{
Christian Rene Leal-Pacheco, Egor A. Maximenko, \\ Gerardo Ramos-Vazquez
}

February 3, 2021

\begin{abstract}
We prove that the homogeneously polyanalytic functions of total order $m$, defined by the system of equations $\bar{D}^{\left(k_{1}, \ldots, k_{n}\right)} f=0$ with $k_{1}+\cdots+k_{n}=m$, can be written as polynomials of total degree $<m$ in variables $\overline{z_{1}}, \ldots, \overline{z_{n}}$, with some analytic coefficients. We establish a weighted mean value property for such functions, using a reproducing property of Jacobi polynomials. After that, we give a general recipe to transform a reproducing kernel by a weighted change of variables. Applying these tools, we compute the reproducing kernel of the Bergman space of homogeneously polyanalytic functions on the unit ball in $\mathbb{C}^{n}$ and on the Siegel domain. For the one-dimensional case, analogous results were obtained by Koshelev (1977), Pessoa (2014), Hachadi and Youssfi (2019).

Mathematical Subject Classification (2020): 32A25, 32K99, 30H20, 46E22, 47B32, 47B37, 33C45.

Keywords: polyanalytic function of several variables, reproducing kernel, mean value property, Bergman space, Jacobi polynomial, Möbius transform, pseudohyperbolic distance.
\end{abstract}

\section{Contents}

1 Introduction $\quad 2$

2 Homogeneously polyanalytic functions 4

$\begin{array}{lll}3 & \text { Weighted mean value property } & 7\end{array}$

4 Pushforward reproducing kernel $\quad 10$

5 Reproducing kernel on the unit ball $\quad 12$

$\begin{array}{lll}6 & \text { Reproducing kernel on the Siegel domain } & 17\end{array}$ 


\section{Introduction}

Bergman [7] comprehensively studied spaces of square-integrable analytic functions on one-dimensional domains, considering them as reproducing kernel Hilbert spaces (RKHS). For some of multidimensional generalizations, see $[11,28,29]$. Polyanalytic functions, have been attracted attention of many mathematicians since the beginning of the 20th century. See some of their properties, applications, and history in $[1,2,5,12,14]$.

Koshelev [16] proved that every integrable $m$-analytic function $f$ on $\mathbb{D}$ fulfills an analog of the mean value property:

$$
f(0)=\frac{1}{\pi} \int_{\mathbb{D}} f(z) P\left(|z|^{2}\right) \mathrm{d} \mu(z),
$$

where $P$ is a certain polynomial of degree $m-1$ with explicitly computed coefficients. Furthermore, he proved that the corresponding space $\mathcal{A}_{m}^{2}(\mathbb{D})$ is a RKHS and gave an explicit formula for the reproducing kernel (RK) at the arbitrary point $z_{0}$ of the disk, using the Möbius transormation $\varphi_{z_{0}}$ that interchanges $z_{0}$ with the origin. Due to the format of the journal, his explanation was extremely short: "although the class of polyanalytic functions is not invariant relative to fractional-linear transformations, this device is still usefull thanks to the presence of $K_{n}\left(z, z_{0}\right)$ under the integral sign". Pessoa [20] identified $P$ with a certain shifted Jacobi polynomial and explained very clearly, how to translate the reproducing property from the origin to an arbitrary point $z_{0}$ of the disk. Namely, he found a correcting factor that restores the polyanalyticity and converts the composition operator $f \mapsto f \circ \varphi_{z_{0}}$ into a unitary operator in $\mathcal{A}_{m}^{2}(\mathbb{D})$. He also computed [19] the RK of the space $\mathcal{A}_{m}^{2}\left(\mathbb{H}_{1}\right)$ of $m$-analytic functions on the upper halfplane $\mathbb{H}_{1}$ in $\mathbb{C}$. Hachadi and Youssfi [13] studied polyanalytic functions on the disk and on the entire complex plane, provided with radial measures. In particular, they computed the RK of $\mathcal{A}_{m}^{2}\left(\mathbb{D}, \mu_{\alpha}\right)$, where $\mathrm{d} \mu_{\alpha}(z)=\frac{1}{\pi}\left(1-|z|^{2}\right)^{\alpha} \mathrm{d} \mu(z)$.

In this paper, we extend some of these results to the unit ball $\mathbb{B}_{n}$ in $\mathbb{C}^{n}$ and to the Siegel domain $\mathbb{H}_{n}:=\left\{\xi \in \mathbb{C}^{n}: \operatorname{Im}\left(\xi_{n}\right)>\left|\xi_{1}\right|^{2}+\cdots+\left|\xi_{n}\right|^{2}\right\}$.

Let $\mathbb{N}=\{1,2, \ldots\}, \mathbb{N}_{0}=\{0,1,2, \ldots\}, n \in \mathbb{N}$. We employ the usual notation for the multi-indices and the notation $|\cdot|$ for the norm in $\mathbb{C}^{n}$, see [25, Section 1.1]. Given an open set $\Omega$ in $\mathbb{C}^{n}$, a multi-index $k=\left(k_{1}, \ldots, k_{n}\right)$ in $\mathbb{N}_{0}^{n}$ and a function of the class $C^{|k|}(\Omega)$,

we denote by $\bar{D}^{k} f$ the Wirtinger derivative of $f$ of the order $k$ (such derivatives were previously used by Poincaré, Pompeiu, and Kolossov). In a more classical notation,

$$
\bar{D}^{k} f(z):=\frac{\partial^{|k|}}{\partial^{k_{1}} \overline{z_{1}} \cdots \partial^{k_{n}} \overline{z_{n}}} f(z) \quad(z \in \Omega) .
$$

Let $\mathcal{A}(\Omega)$ be the class of all analytic functions on $\Omega$. It is defined by the system of equations

$$
\bar{D}^{(1,0, \ldots, 0)} f=0, \quad \bar{D}^{(0,1, \ldots, 0)} f=0, \quad \ldots, \quad \bar{D}^{(0,0, \ldots, 1)} f=0
$$


Given an open subset $\Omega$ of $\mathbb{C}^{n}$ and a multi-index $k=\left(k_{1}, \ldots, k_{n}\right)$ in $\mathbb{N}^{n}, k$-analytic functions on $\Omega$ are defined [5, Section 6.4] as functions that can be represented in the form

$$
f(z)=\sum_{j_{1}, \ldots, j_{n}=0}^{k_{1}-1, \ldots, k_{n}-1} g_{j}(z) \bar{z}^{j}
$$

where all functions $g_{j}$ are analytic. We denote by $\mathcal{A}_{k}(\Omega)$ the class of all functions of the form (1.2). For simply connected domains $\Omega$, such functions can also be characterized as smooth solutions of the system of differential equations

$$
\bar{D}^{\left(k_{1}, 0, \ldots, 0\right)} f=0, \quad \bar{D}^{\left(0, k_{2}, \ldots, 0\right)} f=0, \quad \ldots, \quad \bar{D}^{\left(0,0, \ldots, k_{n}\right)} f=0 .
$$

Instead of considering polyanalytic functions of a given multi-order $k$, we prefer to work with the following classes of "homogeneously polyanalytic" functions.

Definition 1.1. Let $\Omega$ be an open set in $\mathbb{C}^{n}$ and $m \in \mathbb{N}$. We say that $f: \Omega \rightarrow \mathbb{C}$ is homogeneously polyanalytic of total order $m$ or just $m$-analytic, if $f$ belongs to the class $C^{m}(\Omega)$ and $\bar{D}^{k} f=0$ for every $k$ in $\mathbb{N}_{0}^{n}$ with $|k|=m$. We denote by $\mathcal{A}_{m}(\Omega)$ the set of all such functions.

The multi-indices $k$ with $|k|=m$ can be associated with $m$-multisubsets of the set $\{1, \ldots, n\}$, and the number of such multi-indices is $\left(\begin{array}{c}n+m-1 \\ m\end{array}\right)$. For example, the class $\mathcal{A}_{1}(\Omega)=\mathcal{A}(\Omega)$ is defined by $n$ differential equations (1.1).

Definition 1.2. Let $\Omega$ be an open set in $\mathbb{C}^{n}$ and $m \in \mathbb{N}$. We denote by $\widetilde{\mathcal{A}}_{m}(\Omega)$ the set of all functions $f: \Omega \rightarrow \mathbb{C}$ that can be written in the form

$$
f(z)=\sum_{|j|<m} h_{j}(z) \bar{z}^{j}
$$

where $h_{j} \in \mathcal{A}(\Omega)$ for all $j \in \mathbb{N}_{0}^{n}$ with $|j|<m$.

In Section 2 we prove that $\widetilde{\mathcal{A}}_{m}(\Omega)=\mathcal{A}_{m}(\Omega)$. Obviously, $\mathcal{A}_{m}(\Omega)$ is a complex vector space. In Proposition 2.7 we show that the space $\mathcal{A}_{m}(\Omega)$, with $m$ in $\mathbb{N}$, is invariant under linear changes of variables (of course, the domain can change). In a contrast, the spaces $\mathcal{A}_{k}$, with $n \geq 2$ and $k \in \mathbb{N}^{n}, k \neq(1,1, \ldots, 1)$, are not invariant under linear changes of variables; see Proposition 2.8. If $m \in \mathbb{N}$, then $\mathcal{A}_{m}(\Omega) \subseteq \mathcal{A}_{(m, \ldots, m)}(\Omega)$, and some results about $k$-analytic functions $\left(k \in \mathbb{N}^{n}\right)$ can be applied to $\mathcal{A}_{m}(\Omega)$. On the other hand, if $k$ in $\mathbb{N}^{n}$, then $\mathcal{A}_{k}(\Omega) \subseteq \mathcal{A}_{|k|+1-n}(\Omega)$.

From now on, we denote by $\mu$ the Lebesgue measure on $\mathbb{C}^{n}$.

Definition 1.3. Let $\Omega$ be an open set in $\mathbb{C}^{n}, m \in \mathbb{N}, W: \Omega \rightarrow(0,+\infty)$ be a continuous function, and $\mathrm{d} \nu=W \mathrm{~d} \mu$. We denote by $\mathcal{A}_{m}^{2}(\Omega, \nu)$ the set of all functions $f \in \mathcal{A}_{m}(\Omega)$ that are square-integrable with respect to $\nu$. We consider this space with the inner product inherited from $L^{2}(\Omega, \nu)$. Furthermore, we denote by $\mathcal{A}_{(m)}^{2}(\Omega, \nu)$ the orthogonal complement of $\mathcal{A}_{m-1}^{2}(\Omega, \nu)$ in $\mathcal{A}_{m}(\Omega, \nu)$. Here $\mathcal{A}_{0}^{2}(\Omega, \nu):=\{0\}$. 
Section 3 contains a weighted mean-value property for integrable functions belonging to $\mathcal{A}_{m}(\Omega)$. As a consequence of this property, $\mathcal{A}_{m}^{2}(\Omega, \nu)$ is a RKHS. In Section 4 we show how the RK transforms under a weighted change of variables. In Section 5 we use the previous tools to compute the RK of $\mathcal{A}_{m}^{2}\left(\mathbb{B}_{n}, \mu_{\alpha}\right)$, where $\mathbb{B}_{n}$ is the unit ball in $\mathbb{C}^{n}$ and $\mu_{\alpha}$ is the standard radial measure on $\mathbb{B}_{n}$, see (3.13). Finally, in Section 6 we compute the RK of $\mathcal{A}_{m}^{2}\left(\mathbb{H}_{n}, \nu_{\alpha}\right)$, where $\mathbb{H}_{n}$ is the standard Siegel domain in $\mathbb{C}^{n}$ and $\nu_{\alpha}$ is a weighted Lebesgue measure (see (6.1) and (6.2)).

There are many recent investigations on Toeplitz operators, acting in polyanalytic Bergman spaces over one-dimensional domains [8, 15, 17, 18, 24, 27]. We hope that this paper can serve as a basis for some multidimensional generalizations, see Remarks 5.10, 5.11 , and 6.17 .

Finalizing this introduction, we mention several multidimensional results about polyanalytic spaces and kernels in other settings. Askour, Intissar, and Mouayn [4] computed the RK of the space of polyanalytic functions on $\mathbb{C}^{n}$, square-integrable with respect to the Gaussian weight (i.e., the polyanalytic Bargmann-Segal-Fock space). If $k \in \mathbb{N}^{n}$ and $(\Omega, \nu)$ is a direct product of one-dimensional domains with some weights (for example, $\Omega=\mathbb{C}^{n}$ or $\left.\Omega=\mathbb{D}^{n}\right)$, then the RK of $\mathcal{A}_{k}(\Omega, \nu)$ can be obtained as the tensor product of the corresponding reproducing kernels on one-dimensional domains [13]. Ramírez Ortega and Sánchez Nungaray [23] defined some polyanalytic-type spaces on the Siegel domain $\mathbb{H}_{n}$ by other systems of differential equations, involving non-constant coefficients.

\section{Homogeneously polyanalytic functions}

Let $\Omega$ be an open set in $\mathbb{C}^{n}$ and $m \in \mathbb{N}$. In this section we show that $\mathcal{A}_{m}(\Omega)=\widetilde{\mathcal{A}}_{m}(\Omega)$ and mention some other properties of $\mathcal{A}_{m}(\Omega)$.

Lemma 2.1. Let $f \in \mathcal{A}_{m}(\Omega)$. Then the following function is analytic:

$$
g(z):=\sum_{\substack{k \in \mathbb{N}_{0}^{n} \\|k|<m}} \frac{(-1)^{|k|}}{k !}\left(\bar{D}^{k} f\right)(z) \bar{z}^{k} .
$$

Proof. Let $p \in\{1, \ldots, n\}$ and $e_{p}$ be the $p$-th canonical vector in $\mathbb{N}_{0}^{n}$, i.e., $e_{p}:=\left(\delta_{p, s}\right)_{s=1}^{n}$, where $\delta$ is the Kronecker's delta. We have to show that $\bar{D}^{e_{p}} g=0$. By the product rule,

$$
\left(\bar{D}^{e_{p}} g\right)(z)=S_{1}(z)+S_{2}(z)+S_{3}(z)+S_{4}(z)
$$

where

$$
\begin{aligned}
& S_{1}(z)=\sum_{|k|<m-1} \frac{(-1)^{|k|}}{k !}\left(\bar{D}^{k+e_{p}} f\right)(z) \bar{z}^{k}, \quad S_{2}(z)=\sum_{|k|=m-1} \frac{(-1)^{|k|}}{k !}\left(\bar{D}^{k+e_{p}} f\right)(z) \bar{z}^{k}, \\
& S_{3}(z)=\sum_{\substack{|k|<m \\
k_{p}=0}} \frac{(-1)^{|k|}}{k !}\left(\bar{D}^{k} f\right)(z) \bar{D}^{e_{p}}\left(\bar{z}^{k}\right), \quad S_{4}(z)=\sum_{\substack{|k|<m \\
k_{p}>0}} \frac{(-1)^{|k|}}{\left(k-e_{p}\right) !}\left(\bar{D}^{k} f\right)(z) \bar{z}^{k-e_{p}} .
\end{aligned}
$$


We have that $S_{2}(z)=0$, because $f \in \mathcal{A}_{m}(\Omega)$ and $\left|k+e_{p}\right|=m$ in the sum defining $S_{2}$. Also $S_{3}(z)=0$, because $\bar{D}^{e_{p}} \bar{z}^{k}=0$ when $k_{p}=0$. Finally, with the change of variable $j=k-e_{p}$, we rewrite $S_{4}(z)$ as

$$
S_{4}(z)=-\sum_{|j|<m-1} \frac{(-1)^{|j|}}{j !}\left(\bar{D}^{r+e_{p}} f\right)(z) \bar{z}^{j} .
$$

Therefore, $\left(\bar{D}^{e_{p}} g\right)(z)=S_{1}(z)+S_{4}(z)=0$.

Lemma 2.2. Let $f \in \widetilde{\mathcal{A}}_{p}(\Omega)$ and $g \in \widetilde{\mathcal{A}}_{q}(\Omega)$. Then $f g \in \widetilde{\mathcal{A}}_{p+q-1}(\Omega)$.

Proof. This lemma follows from the elementary observation that if $j \in \mathbb{N}_{0}^{n}$ and $k \in \mathbb{N}_{0}^{n}$, with $|j|<p$ and $|k|<q$, then $\bar{z}^{j} \bar{z}^{k}=\bar{z}^{j+k}$ and $|j+k|=|j|+|k|<p+q-1$.

Theorem 2.3. Let $\Omega$ be an open set in $\mathbb{C}^{n}$ and $m \in \mathbb{N}$. Then $\mathcal{A}_{m}(\Omega)=\widetilde{\mathcal{A}}_{m}(\Omega)$.

Proof. It is well known that $\mathcal{A}(\Omega)=\widetilde{\mathcal{A}}(\Omega)$. Let $m>1$. It is obvious that $\widetilde{\mathcal{A}}_{m}(\Omega) \subseteq$ $\mathcal{A}_{m}(\Omega)$. We show, by induction on $m$, that $\mathcal{A}_{m}(\Omega) \subseteq \widetilde{\mathcal{A}}_{m}(\Omega)$. Suppose $\mathcal{A}_{p}(\Omega) \subseteq \widetilde{\mathcal{A}}_{p}(\Omega)$ for every $p<m$ and let $f \in \mathcal{A}_{m}(\Omega)$. Define $g$ as in Lemma 2.1, then observe that

$$
f(z)=-\sum_{0<|k|<m} \frac{(-1)^{|k|}}{k !}\left(\bar{D}^{k} f\right)(z) \bar{z}^{k}+g(z) .
$$

For every $k$ with $0<|k|<m$, we have $\bar{z}^{k} \in \widetilde{\mathcal{A}}_{|k|+1}(\Omega)$ and $\bar{D}^{k} f \in \mathcal{A}_{m-|k|}(\Omega) \subseteq \widetilde{\mathcal{A}}_{m-|k|}(\Omega)$; the last inclusion holds by the induction hypothesis. Finally, apply Lemma 2.2.

Corollary 2.4. Let $f \in \mathcal{A}_{m}(\Omega)$ and $a \in \Omega$. Then there exists a family of functions $\left(h_{k}\right)_{|k|<m}$ in $\mathcal{A}(\Omega)$, such that for every $z$ in $\Omega$,

$$
f(z)=\sum_{\substack{k \in \mathbb{N}_{0}^{n} \\|k|<m}} h_{k}(z)(\bar{z}-\bar{a})^{k} .
$$

Proof. First, we write $f$ as (1.4). Then, expanding $\bar{z}^{j}=(\bar{z}-\bar{a}+\bar{a})^{j}$ into multi-powers of $\bar{z}-\bar{a}$ and regrouping the summands, we obtain a sum of the form (2.1).

Corollary 2.5. Let $f \in \mathcal{A}_{m}(\Omega), a \in \Omega$, and $r>0$ such that $a+r \mathbb{B}_{n} \subseteq \Omega$. Then there exists a family $(\beta)_{j, k \in \mathbb{N}_{0}^{n},|k|<m}$ of complex numbers such that for every $z$ in $a+r \mathbb{B}_{n}$,

$$
f(z)=\sum_{j \in \mathbb{N}_{0}^{n}} \sum_{\substack{k \in \mathbb{N}_{0}^{n} \\|k|<m}} \beta_{j, k}(z-a)^{j}(\bar{z}-\bar{a})^{k} .
$$

Moreover, this series converges uniformly on every compact subset of $\mathbb{B}_{n}$. 
Proof. It is well known that every holomorphic function on $\mathbb{B}_{n}$, decomposes on $\mathbb{B}_{n}$ into a power series, converging on $\mathbb{B}_{n}$ and uniformly converging on compact subsets of $\mathbb{B}_{n}$. Applying this fact to each $h_{j}$ from Corollary 2.4, we obtain (2.2).

Let us mention a version of the uniqueness property for $m$-analytic functions.

Proposition 2.6. Let $\Omega$ be a connected open set in $\mathbb{C}^{n}, \Omega_{1}$ be an open subset of $\Omega$, and $f \in \mathcal{A}_{m}(\Omega)$ such that $f(z)=0$ for every $z$ in $\Omega_{1}$. Then $f(z)=0$ for every $z$ in $\Omega$.

Proof. For $k$ in $\mathbb{N}^{n}$, the uniqueness property of $k$-analytic functions is proven in [5, Section 6.4]. The uniqueness property for $m$-analytic functions is a corollary of this fact, since $\mathcal{A}_{m}(\Omega) \subseteq \mathcal{A}_{(m, \ldots, m)}(\Omega)$.

To finish this section, we will show that the class $\mathcal{A}_{m}$ with $m$ in $\mathbb{N}$ is closed under linear changes of variables, while the classes $\mathcal{A}_{k}$ with $k \in \mathbb{N}^{n}$ are generally not.

Proposition 2.7. Let $M$ be an invertible $n \times n$ complex matrix and $f$ in $\mathcal{A}_{m}(\Omega)$. Define $g: M \Omega \rightarrow \mathbb{C}$ by $g(z):=f\left(M^{-1} z\right)$. Then $g \in \mathcal{A}_{m}(M \Omega)$.

Proof. Theorem 2.3 allows us to work with $\widetilde{\mathcal{A}}_{m}$ instead of $\mathcal{A}_{m}$. Let $f$ be like in Definition 1.2. Then

$$
g(z)=\sum_{|j|<m} h_{j}\left(M^{-1} z\right){\overline{\left(M^{-1} z\right)}}^{j} .
$$

The functions $z \mapsto h_{j}\left(M^{-1} z\right)$ are analytic. Let $M^{-1}=\left[c_{r, s}\right]_{r, s=1}^{n}$. Then

$$
{\overline{\left(M^{-1} z\right)}}^{j}=\prod_{r=1}^{n}\left(\sum_{s=1}^{n} \overline{c_{r, s}} \overline{z_{s}}\right)^{j_{r}} .
$$

The last expression is a homogeneous polynomial in $\overline{z_{1}}, \ldots, \overline{z_{n}}$ of total degree $|j|$, which is strictly less than $m$ (the same conclusion can also be obtained by Lemma 2.2). Therefore $g \in \widetilde{\mathcal{A}}_{m}(M \Omega)$.

Proposition 2.8. Let $n \geq 2, \Omega$ be an open subset of $\mathbb{C}^{n}, k \in \mathbb{N}^{n}, k \neq(1,1, \ldots, 1)$. Then there exists a function $f$ in $\mathcal{A}_{k}(\Omega)$ and an invertible matrix $M$ in $\mathbb{C}^{n \times n}$ such that the function $g: M \Omega \rightarrow \mathbb{C}$, defined by $g(z):=f\left(M^{-1} z\right)$, does not belong to $\mathcal{A}_{k}(M \Omega)$.

Proof. To simplify the notation, we suppose that $k_{1}>1$. The general case is analogous. Define $M$ in such a manner that

$$
M^{-1} z=\left(z_{1}+z_{2}, z_{2}-z_{1}, z_{3}, \ldots, z_{n}\right) .
$$

Consider $f: \Omega \rightarrow \mathbb{C}, f(z):={\overline{z_{1}}}^{k_{1}-1}{\overline{z_{2}}}^{k_{2}-1}$. Then

$$
g(z)=\left(\overline{z_{1}}+\overline{z_{2}}\right)^{k_{1}-1}\left(\overline{z_{2}}-\overline{z_{1}}\right)^{k_{2}-1} .
$$

In the expansion of the last polynomial, one of the terms is $\bar{z}_{2} k_{1}+k_{2}-2$. Since $k_{1}+k_{2}-2>$ $k_{2}-1$, we obtain $g \notin \mathcal{A}_{k}(M \Omega)$, though $f \in \mathcal{A}_{k}(\Omega)$. 


\section{$3 \quad$ Weighted mean value property}

In this section we prove that the value of a $m$-analytic function at the center of the unit ball $\mathbb{B}_{n}$ can be expressed as the integral of this function over the ball, with a certain real radial weight (Theorem 3.3). Similar results in the one-dimensional case were proved in $[13,16,20]$.

\section{Jacobi polynomials and their reproducing property}

Some integrals over the unit ball, written in the spherical coordinates, reduce to integrals over the unit interval $(0,1)$ with weights of power type at the boundary points 0 and 1 . Thereby Jacobi polynomials naturally appear. They can be defined by Rodrigues formula:

$$
P_{m}^{(\xi, \eta)}(x):=\frac{(-1)^{m}}{2^{m} m !}(1-x)^{-\xi}(1+x)^{-\eta} \frac{\mathrm{d}^{m}}{\mathrm{~d} x^{m}}\left((1-x)^{m+\xi}(1+x)^{m+\eta}\right) .
$$

Here are well-known explicit formulas for $P_{m}$ :

$$
\begin{aligned}
P_{m}^{(\xi, \eta)}(x) & =\sum_{s=0}^{m}\left(\begin{array}{c}
\xi+\eta+m+s \\
s
\end{array}\right)\left(\begin{array}{c}
\xi+m \\
m-s
\end{array}\right)\left(\frac{x-1}{2}\right)^{s} \\
& =\sum_{s=0}^{m}(-1)^{s}\left(\begin{array}{c}
\xi+\eta+m+s \\
s
\end{array}\right)\left(\begin{array}{l}
\eta+m \\
m-s
\end{array}\right)\left(\frac{x+1}{2}\right)^{s} .
\end{aligned}
$$

If $\xi, \eta>-1$, then $\left(P_{m}^{(\xi, \eta)}\right)_{m=0}^{\infty}$ is an orthogonal family on the interval $(-1,1)$ with respect to the weight $(1-x)^{\xi}(1+x)^{\eta}$. Using (3.1) and integrating by parts yields the following integral formula:

$$
\int_{-1}^{1} P_{m}^{(\alpha, \beta+1)}(x)(1-x)^{\alpha}(1+x)^{\beta} \mathrm{d} x=2^{\alpha+\beta+1}(-1)^{m} \mathrm{~B}(\alpha+m+1, \beta+1) .
$$

Definition 3.1. Let $m \in \mathbb{N}$ and $\alpha, \beta>-1$. We denote by $R_{m}^{(\alpha, \beta)}$ the following polynomial:

$$
R_{m}^{(\alpha, \beta)}(t):=\frac{(-1)^{m} \mathrm{~B}(\alpha+1, \beta+1)}{\mathrm{B}(\alpha+m+1, \beta+1)} P_{m}^{(\alpha, \beta+1)}(2 t-1) .
$$

Equivalently, by the symmetry relation for Jacobi polynomials,

$$
R_{m}^{(\alpha, \beta)}(t)=\frac{\mathrm{B}(\alpha+1, \beta+1)}{\mathrm{B}(\alpha+m+1, \beta+1)} P_{m}^{(\beta+1, \alpha)}(1-2 t) .
$$

Combining (3.5) with (3.3) or (3.6) with (3.2), we get more explicit formulas for $R_{m}^{(\alpha, \beta)}$ :

$$
\begin{aligned}
R_{m}^{(\alpha, \beta)}(t) & =\frac{\Gamma(\alpha+1) \Gamma(\beta+m+2)}{\Gamma(\alpha+\beta+2) \Gamma(\alpha+m+1)} \sum_{s=0}^{m} \frac{(-1)^{s} \Gamma(\alpha+\beta+m+s+2)}{s !(m-s) ! \Gamma(\beta+s+2)} t^{s} \\
& =\frac{\Gamma(\alpha+1) \Gamma(\beta+m+2)}{\Gamma(\alpha+\beta+2)(\alpha+m) m !} \sum_{s=0}^{m} \frac{(-1)^{s}\left(\begin{array}{c}
m \\
s
\end{array}\right)}{\mathrm{B}(\alpha+m, \beta+s+2)} t^{s} .
\end{aligned}
$$


The next simple result was proven in [6] using the orthogonality of the Jacobi polynomials and formula (3.4). Previously, Hachadi and Youssfi [13, formula (5.7)] gave another proof for the case $\beta=0$.

Proposition 3.2. Let $m \in \mathbb{N}$ and $\alpha, \beta>-1$. Then for every univariate polynomial $h$ with complex coefficients and $\operatorname{deg}(h) \leq m$,

$$
\frac{1}{\mathrm{~B}(\alpha+1, \beta+1)} \int_{0}^{1} h(t) R_{m}^{(\alpha, \beta)}(t)(1-t)^{\alpha} t^{\beta} \mathrm{d} t=h(0) .
$$

The polynomials of degree $\leq m$, considered as square-integrable functions on the interval $(0,1)$ with the normalized weight $\frac{1}{\mathrm{~B}(\alpha+1, \beta+1)}(1-t)^{\alpha} t^{\beta}$, form a RKHS. Formula (3.9) means that $R_{m}^{(\alpha, \beta)}$ is the RK of this space at the point 0 .

As a particular case of (3.9), for every $k$ in $\mathbb{N}_{0}$ with $k \leq m$,

$$
\frac{1}{\mathrm{~B}(\alpha+1, \beta+1)} \int_{0}^{1} R_{m}^{(\alpha, \beta)}(t)(1-t)^{\alpha} t^{\beta+k} \mathrm{~d} t=\delta_{k, 0} .
$$

\section{Weighted mean value property of homogeneously polyanalytic functions}

We denote by $\mu$ the Lebesgue measure on $\mathbb{C}^{n}$, by $\mathbb{S}_{n}$ the unit sphere in $\mathbb{C}^{n}$, and by $\mu_{\mathbb{S}_{n}}$ the (non-normalized) area measure on $\mathbb{S}_{n}$. It is well known [25, Section 1.4] that

$$
\mu\left(\mathbb{B}_{n}\right)=\frac{\pi^{n}}{n !}, \quad \mu_{\mathbb{S}_{n}}\left(\mathbb{S}_{n}\right)=\frac{2 \pi^{n}}{(n-1) !},
$$

and

$$
\int_{\mathbb{S}_{n}} \zeta^{j} \bar{\zeta}^{k} \mathrm{~d} \mu_{\mathbb{S}_{n}}(\zeta)=\frac{2 \pi^{n} j !}{(n-1+|j|) !} \cdot \delta_{j, k} \quad\left(j, k \in \mathbb{N}_{0}^{n}\right) .
$$

Given an integrable function $f$ on $\mathbb{B}_{n}$, its integral over $\mathbb{B}_{n}$ can be written as

$$
\int_{\mathbb{B}_{n}} f \mathrm{~d} \mu=\int_{0}^{1} r^{2 n-1}\left(\int_{\mathbb{S}_{n}} f(r \zeta) \mathrm{d} \mu_{\mathbb{S}_{n}}(\zeta)\right) \mathrm{d} r .
$$

For $\alpha>-1$, we denote by $\mu_{\alpha}$ the Lebesgue measure on $\mathbb{B}_{n}$ with the standard radial weight:

$$
\mathrm{d} \mu_{\alpha}(z)=c_{\alpha}\left(1-|z|^{2}\right)^{\alpha} \mathrm{d} \mu(z) .
$$

The normalizing constant $c_{\alpha}$ is chosen so that $\mu_{\alpha}\left(\mathbb{B}_{n}\right)=1$ :

$$
c_{\alpha}:=\frac{\Gamma(n+\alpha+1)}{\pi^{n} \Gamma(\alpha+1)} .
$$


Theorem 3.3. Let $f \in \mathcal{A}_{m}\left(\mathbb{B}_{n}\right)$ such that $f \in L^{1}\left(\mathbb{B}_{n}, \mu_{\alpha}\right)$. Then

$$
f(0)=\int_{\mathbb{B}_{n}} f(z) R_{m-1}^{(\alpha, n-1)}\left(|z|^{2}\right) \mathrm{d} \mu_{\alpha}(z) .
$$

Proof. We represent $f$ in the form (2.2) with $a=0$, then make the change of variables $z=r \zeta$ with $0 \leq r<1, \zeta \in \mathbb{S}_{n}$ :

$$
f(z)=\sum_{j \in \mathbb{N}_{0}^{n}} \sum_{\substack{k \in \mathbb{N}_{0}^{n} \\|k|<m}} \beta_{j, k} r^{|j|+|k|} \zeta^{j} \bar{\zeta}^{k} .
$$

For every $s$ in $(0,1)$, let $I_{s}$ be the integral similar to the right-hand side of $(3.15)$, but over the ball $s \mathbb{B}_{n}$ :

$$
I_{s}:=\int_{s \mathbb{B}_{n}} f(z) R_{m-1}^{(\alpha, n-1)}\left(|z|^{2}\right) \mathrm{d} \mu_{\alpha}(z) .
$$

Since the series (3.16) converges uniformly over $r$ in $[0, s]$ and $\zeta$ in $\mathbb{S}_{n}$, it can be interchanged with the integral over $s \mathbb{B}_{n}$. Then we apply (3.12) and (3.11):

$$
\begin{aligned}
I_{s} & =c_{\alpha} \sum_{j \in \mathbb{N}_{0}^{n}} \sum_{\substack{k \in \mathbb{N}_{0}^{n} \\
|k|<m}} \beta_{j, k} \int_{0}^{s} r^{2 n-1+|j|+|k|} R_{m-1}^{(\alpha, n-1)}\left(r^{2}\right)\left(1-r^{2}\right)^{\alpha}\left(\int_{\mathbb{S}_{n}} \zeta^{j} \bar{\zeta}^{k} \mathrm{~d} \mu_{\mathbb{S}_{n}}(\zeta)\right) \mathrm{d} r \\
& =c_{\alpha} \sum_{\substack{k \in \mathbb{N}_{0}^{n} \\
|k|<m}} \beta_{k, k} \cdot \frac{\pi^{n} k !}{(n-1+|k|) !} \int_{0}^{s} R_{m-1}^{(\alpha, n-1)}(t)(1-t)^{\alpha} t^{n-1+|k|} \mathrm{d} t .
\end{aligned}
$$

The condition $f \in L^{1}\left(\mathbb{B}_{n}, \mu_{\alpha}\right)$ implies that $I_{s} \rightarrow I_{1}$, as $s \rightarrow 1$. Passing to this limit and using (3.10), we finally obtain

$$
\begin{aligned}
I_{1} & =\frac{\Gamma(n+\alpha+1)}{\Gamma(\alpha+1)} \sum_{\substack{k \in \mathbb{N}_{0}^{n} \\
|k|<m}} \beta_{k, k} \cdot \frac{k !}{(n-1+|k|) !} \int_{0}^{1} R_{m-1}^{(\alpha, n-1)}(t)(1-t)^{\alpha} t^{n-1+|k|} \mathrm{d} t \\
& =\frac{\Gamma(\alpha+n+1)}{\Gamma(\alpha+1)} \sum_{|k|<m} \beta_{k, k} \frac{k !}{(n-1+|k|) !} \cdot \delta_{k, 0} \mathrm{~B}(\alpha+1, n)=\beta_{0,0}=f(0) .
\end{aligned}
$$

Here is an analog of (3.15) for an arbitrary ball and for $\alpha=0$.

Corollary 3.4. Let $\Omega$ be an open subset of $\mathbb{C}^{n}, f \in \mathcal{A}_{m}(\Omega), a \in \Omega$, and $r>0$ such that $a+r \mathbb{B}_{n} \subseteq \Omega$. Suppose that $f \in L^{1}\left(a+r \mathbb{B}_{n}, \mu\right)$. Then

$$
f(a)=\frac{n !}{\pi^{n}} \frac{1}{r^{2 n}} \int_{a+r \mathbb{B}_{n}} f(z) R_{m-1}^{(0, n-1)}\left(\frac{|z-a|^{2}}{r^{2}}\right) \mathrm{d} \mu(z) .
$$




\section{Bergman spaces of homogeneously polyanalytic functions}

In the rest of this section, we suppose that $\Omega, m, W, \nu$ are like in Definition 1.3. Using (3.17), it is easy to prove the upcoming Lemma 3.5 and Proposition 3.6. See similar proofs for the one-dimensional case in [6, Lemma 4.3, Proposition 4.4].

Lemma 3.5. Let $K$ be a compact subset of $\Omega$. Then there exists a number $C_{m, W, K}>0$ such that for every $f$ in $\mathcal{A}_{m}^{2}(\Omega, \nu)$ and every $z$ in $K$,

$$
|f(z)| \leq C_{m, W, K}\|f\|_{\mathcal{A}_{m}^{2}(\Omega, \nu)} .
$$

Proposition 3.6. $\mathcal{A}_{m}^{2}(\Omega, \nu)$ is a $R K H S$.

As a corollary, the spaces $\mathcal{A}_{(m)}^{2}(\Omega, \nu)$ are also RKHS.

Proposition 3.7. In the conditions of Definition 1.3, suppose additionally that $\Omega$ is bounded and $\nu$ is finite. Then

$$
L^{2}(\Omega, \nu)=\bigoplus_{m=1}^{\infty} \mathcal{A}_{(m)}^{2}(\Omega, \nu) .
$$

Proof. This is a simple consequence of three facts: 1) the continuous functions with compact supports form a dense subset of $L^{2}(\Omega, \nu)$; 2) by the Stone-Weierstrass theorem, every continuous function on the closure of $\Omega$ can be uniformly approximated by polynomials in $z_{1}, \ldots, z_{n}, \overline{z_{1}}, \ldots, \overline{z_{n}}$; and 3$)$ the norm of $L^{2}(\Omega, \nu)$ can be estimated from above by a constant multiple of the maximum-norm.

In the one-dimensional case, the "true- $m$-analytic" spaces $\mathcal{A}_{(m)}^{2}$ were studied by Ramazanov [22] and Vasilevski [26,27]. According to [26] (see also another proof in [18]), the decomposition (3.19) holds for the poly-Fock space $\mathcal{A}_{m}^{2}\left(\mathbb{C}, \mathrm{e}^{-|z|^{2}} \mathrm{~d} \mu\right)$. On the other hand, if $\Omega$ is the upper halfplane $\mathbb{H}_{1}$ with the Lebesgue measure, then $L^{2}\left(\mathbb{H}_{1}\right)$ decomposes into the orthogonal sum of the spaces $\mathcal{A}_{(m)}^{2}\left(\mathbb{H}_{1}\right)$ and their conjugates [27, Theorem 3.3.5], and (3.19) fails. It is natural to ask if Proposition 3.7 remains true if $\nu(\Omega)<+\infty$, without assuming $\Omega$ to be bounded.

\section{Pushforward reproducing kernel}

In this section we show how to transform a RK using a weighted change of variables. First, we deal with abstract positive kernels [3], then we consider reproducing kernels in Hilbert spaces.

Let $X$ be a non-empty set. We denote by $\mathbb{C}^{X}$ the complex vector space of all functions $X \rightarrow \mathbb{C}$ with pointwise operations. A family $\left(K_{x}\right)_{x \in X}$ with values in $\mathbb{C}^{X}$ is called a positive kernel on $X$ if for every $m$ in $\mathbb{N}$, every $x_{1}, \ldots, x_{m}$ in $X$ and every $\alpha_{1}, \ldots, \alpha_{m}$ in $\mathbb{C}$,

$$
\sum_{r, s=1}^{m} \alpha_{r} \overline{\alpha_{s}} K_{x_{r}}\left(x_{s}\right) \geq 0 .
$$


Proposition 4.1. Let $X, Y$ be non-empty sets, $\psi: Y \rightarrow X$ and $J: Y \rightarrow \mathbb{C}$ be some functions, and $\left(K_{x}\right)_{x \in X}$ be a positive kernel on $X$. Then the family $\left(L_{u}\right)_{u \in Y}$, defined by

$$
L_{u}(v):=\overline{J(u)} J(v) K_{\psi(u)}(\psi(v))
$$

is a positive kernel on $Y$.

Proof. Let $m \in \mathbb{N}, u_{1}, \ldots, u_{m} \in Y, \alpha_{1}, \ldots, \alpha_{m} \in \mathbb{C}$. For every $s$ in $\{1, \ldots, m\}$ put $x_{s}:=\psi\left(u_{s}\right)$ and $\beta_{s}:=\overline{J\left(u_{s}\right)} \alpha_{s}$. Then

$$
\sum_{r, s=1}^{m} \alpha_{r} \overline{\alpha_{s}} L_{u_{r}}\left(u_{s}\right)=\sum_{r, s=1}^{m} \beta_{r} \overline{\beta_{s}} K_{x_{r}}\left(x_{s}\right) \geq 0 .
$$

Let $X$ be a non-empty set. We say that $H$ is a Hilbert space of functions on $X$ if $H$ is a vector subspace of $\mathbb{C}^{X}$, provided with an inner product and complete with respect to the corresponding norm. Furthermore, if $x \in X, \mathcal{K} \in H$ and $\langle f, \mathcal{K}\rangle=f(x)$ for every $f$ in $H$, then we say that $\mathcal{K}$ is a reproducing kernel of $H$ at the point $x$. In case of existence, this function is unique.

Proposition 4.2. Let $X, Y$ be non-empty sets, $\psi: Y \rightarrow X$ and $J: Y \rightarrow \mathbb{C}$ be some functions, $H_{1}$ be a Hilbert space of functions over $X, H_{2}$ be a Hilbert space of functions over $Y$, and

$$
(U f)(z):=J(z) f(\psi(z))
$$

be a well-defined unitary operator mapping $H_{1}$ onto $H_{2}$. Suppose that $u \in Y$ and $\mathcal{K}$ be the reproducing kernel of $H_{1}$ at the point $\psi(u)$. Then the function $\mathcal{L}: Y \rightarrow \mathbb{C}$, defined by the following rule, is the reproducing kernel of $\mathrm{H}_{2}$ at the point $u$ :

$$
\mathcal{L}(v):=\overline{J(u)} J(v) \mathcal{K}(\psi(v)) .
$$

Proof. Let $g \in H_{2}$ and $f=U^{-1} g$. Then

$$
g(u)=J(u) f(\psi(u))=J(u)\langle f, \mathcal{K}\rangle_{H_{1}}=\langle g, \overline{J(u)} U \mathcal{K}\rangle_{H_{2}} .
$$

Defining $\mathcal{L}$ by $\mathcal{L}(v)=\overline{J(u)}(U \mathcal{K})(v)=\overline{J(u)} J(v) \mathcal{K}(\psi(v))$, we get the RK of $H_{2}$ at $u$.

Proposition 4.3. Let $X, Y$ be non-empty sets, $\psi: Y \rightarrow X$ and $J: Y \rightarrow \mathbb{C}$ be some functions, $H_{1}$ be a Hilbert space of functions over $X$ with reproducing kernel $\left(K_{x}\right)_{x \in X}, H_{2}$ be a Hilbert space of functions over $Y$, and

$$
(U f)(z):=J(z) f(\psi(z))
$$

be a well-defined unitary operator mapping $H_{1}$ onto $H_{2}$. Then $H_{2}$ is a RKHS, and its reproducing kernel $\left(L_{u}\right)_{u \in Y}$ is given by

$$
L_{u}(v)=\overline{J(u)} J(v) K_{\psi(u)}(\psi(v)) .
$$


Proof. Apply Proposition 4.2 at every point $u$ of $Y$.

As a simple application of the this scheme, let us express the Berezin transform in $H_{2}$ via the Berezin transform in $H_{1}$. Given a Hilbert space $H$, we denote by $\mathcal{B}(H)$ the $\mathrm{C}^{*}$-algebra of all bounded linear operators acting in $H$. Given a set $X$, we denote by $B(X)$ the Banach space of all bounded functions on $X$, with the supremum norm. If $H$ is a RKHS over $X$ and its RK satisfies $\left\|K_{x}\right\|_{H} \neq 0$ for every $x$ in $X$, then the Berezin transform $\operatorname{Ber}_{H}: \mathcal{B}(H) \rightarrow B(X)$ is defined by

$$
\operatorname{Ber}_{H}(A)(x):=\frac{\left\langle A K_{x}, K_{x}\right\rangle_{H}}{\left\langle K_{x}, K_{x}\right\rangle_{H}} \quad(A \in \mathcal{B}(H), x \in X) .
$$

Proposition 4.4. In the conditions of Proposition 4.3, suppose that $\left\|K_{x}\right\|_{H_{1}} \neq 0$ for every $x$ in $X$ and $J(u) \neq 0$ for every $u$ in $Y$. Then

$$
\operatorname{Ber}_{H_{2}}(A)(u)=\operatorname{Ber}_{H_{1}}\left(U^{*} A U\right)(\psi(u)) \quad\left(A \in \mathcal{B}\left(H_{2}\right), u \in Y\right) .
$$

Proof. As we have seen in Proposition 4.2, $L_{u}(v)=\overline{J(u)}\left(U K_{\psi(u)}\right)(v)$. Therefore,

$$
\begin{aligned}
\operatorname{Ber}_{H_{2}}(A)(u) & =\frac{\left\langle A L_{u}, L_{u}\right\rangle_{H_{2}}}{\left\|L_{u}\right\|^{2}}=\frac{|J(u)|^{2}\left\langle A U K_{\psi(u)}, U K_{\psi(u)}\right\rangle_{H_{2}}}{|J(u)|^{2}\left\|U K_{\psi(u)}\right\|^{2}} \\
& =\frac{\left\langle U^{*} A U K_{\psi(u)}, K_{\psi(u)}\right\rangle_{H_{1}}}{\left\|K_{\psi(u)}\right\|^{2}}=\operatorname{Ber}_{H_{1}}\left(U^{*} A U\right)(\psi(u)) .
\end{aligned}
$$

Corollary 4.5. In the conditions of Proposition 4.4, suppose that $\mathrm{Ber}_{H_{1}}$ is injective. Then $\mathrm{Ber}_{\mathrm{H}_{2}}$ is also injective. Moreover, if $\psi$ is a bijection, than the injectivity of $\operatorname{Ber}_{H_{1}}$ is equivalent to the injectivity of $\mathrm{Ber}_{\mathrm{H}_{2}}$.

\section{$5 \quad$ Reproducing kernel on the unit ball}

In this section we consider the domain $\Omega=\mathbb{B}_{n}$ with the standard radial measure $\mu_{\alpha}$, given by (3.13). Using the weighted mean value property and appropriate unitary operators, we compute the RK of $\mathcal{A}_{m}^{2}\left(\mathbb{B}_{n}, \mu_{\alpha}\right)$.

\section{On the unit ball biholomorphisms}

For a fixed $a$ in $\mathbb{B}_{n} \backslash\{0\}$, we denote by $\varphi_{a}$ the function $\mathbb{B}_{n} \rightarrow \mathbb{B}_{n}$, defined by

$$
\varphi_{a}(z):=\frac{a-\frac{\langle z, a\rangle}{\langle a, a\rangle} a-\sqrt{1-|a|^{2}}\left(z-\frac{\langle z, a\rangle}{\langle a, a\rangle} a\right)}{1-\langle z, a\rangle} .
$$


For $a=0, \varphi_{a}(z):=z$. It is well known [25, Theorem 2.2.2] that for every $a$ in $\mathbb{B}_{n}, \varphi_{a}$ is a biholomorphism of $\mathbb{B}_{n}, \varphi_{a}\left(\varphi_{a}(z)\right)=z$ for every $z$ in $\mathbb{B}_{n}, \varphi_{a}(0)=a, \varphi_{a}(a)=0$, and

$$
1-\left\langle\varphi_{a}(z), \varphi_{a}(w)\right\rangle=\frac{(1-\langle a, a\rangle)(1-\langle z, w\rangle)}{(1-\langle z, a\rangle)(1-\langle a, w\rangle)} .
$$

Here are particular cases of (5.2), with $w=z$ and $w=0$, respectively:

$$
\begin{aligned}
1-\left|\varphi_{a}(z)\right|^{2} & =\frac{\left(1-|a|^{2}\right)\left(1-|z|^{2}\right)}{|1-\langle z, a\rangle|^{2}}, \\
1-\left\langle\varphi_{a}(z), a\right\rangle & =\frac{1-|a|^{2}}{1-\langle z, a\rangle} .
\end{aligned}
$$

The real Jacobian of $\varphi_{a}$ is [29, Lemma 1.7]

$$
\left(J_{\mathbb{R}} \varphi_{a}\right)(z)=\left(\frac{1-|a|^{2}}{|1-\langle z, a\rangle|^{2}}\right)^{n+1} .
$$

We denote by $\rho_{\mathbb{B}_{n}}(z, w)$ the expression $\left|\varphi_{z}(w)\right|$, known as the pseudohyperbolic distance between $z$ and $w$, see [29, Corollary 1.22] or [9]. Formula (5.3) provides a simple recipe to compute $\rho_{\mathbb{B}_{n}}(z, w)$.

\section{A factor to preserve the polyanalyticity}

Definition 5.1. Given $a$ in $\mathbb{B}_{n}$, we define $p_{m, a}: \mathbb{B}_{n} \rightarrow \mathbb{C}$ by

$$
p_{m, a}(z):=\left(\frac{1-\langle a, z\rangle}{1-\langle z, a\rangle}\right)^{m-1} .
$$

In the one-dimensional case, the function $p_{m, a}$ was introduced and studied by Pessoa [20]. As it is shown in the proof of Lemma 5.3, the main purpose of $p_{m, a}$ is to eliminate the denominators in the multi-powers of $\overline{\varphi_{a}(z)}$.

Lemma 5.2. For every $a, z$ in $\mathbb{B}_{n}$,

$$
\begin{gathered}
\left|p_{m, a}(z)\right|=1, \\
p_{m, a}\left(\varphi_{a}(z)\right) p_{m, a}(z)=1 .
\end{gathered}
$$

Proof. Formula (5.6) follows directly from the definition of $p_{m, a}$. Identity (5.7) is easy to verify using (5.4).

Lemma 5.3. Let $a \in \mathbb{B}_{n}$ and $f \in \mathcal{A}_{m}\left(\mathbb{B}_{n}\right)$. Then $\left(f \circ \varphi_{a}\right) \cdot p_{m, a} \in \mathcal{A}_{m}\left(\mathbb{B}_{n}\right)$. 
Proof. Let $f$ be of the form (1.4). Denote by $N_{a}(z)$ the numerator of (5.1); it is a polynomial of degree 1 in $z_{1}, \ldots, z_{n}$. Then,

$$
\begin{aligned}
f\left(\varphi_{a}(z)\right) p_{m, \alpha}(z) & =\left(\frac{1-\langle a, z\rangle}{1-\langle z, a\rangle}\right)^{m-1} \sum_{|j|<m} h_{j}\left(\varphi_{a}(z)\right) \frac{{\overline{N_{a}(z)}}^{j}}{(1-\langle a, z\rangle)^{|j|}} \\
& =\sum_{|j|<m} \frac{h_{j}\left(\varphi_{a}(z)\right)}{(1-\langle z, a\rangle)^{m-1}}{\overline{N_{a}(z)}}^{j}(1-\langle a, z\rangle)^{m-1-|j|} .
\end{aligned}
$$

The quotients in the last sum are analytic functions of $z$. The multi-power ${\overline{N_{a}(z)}}^{j}$ is a polynomial in $\overline{z_{1}}, \ldots, \overline{z_{n}}$ of total degree $|j|$, and the expression $(1-\langle a, z\rangle)^{m-1-|j|}$ is a polynomial in $\overline{z_{1}}, \ldots, \overline{z_{n}}$ of total degree $m-1-|j|$. Therefore, the whole sum is a polynomial in $\overline{z_{1}}, \ldots, \overline{z_{m}}$ of total degree at most $m-1$, with some analytic coefficients.

\section{A factor to preserve the norm}

Remark 5.4. In the upcoming formula for $g_{\alpha, a}$ and in some other formulas of this paper, we work with (non necesarily integer) powers of complex numbers. Given $t$ in $\mathbb{C} \backslash\{0\}$ and $\beta$ in $\mathbb{C}$, we define $t^{\beta}$ as $\exp (\beta \log (t))$, where $\log (t)=\log _{\mathbb{R}}|t|+\mathrm{i} \arg (t), \log _{\mathbb{R}}|t|$ is the real $\operatorname{logarithm}$ of $|t|$, and $\arg (t)$ is the principal argument of $t$, belonging to $(-\pi, \pi]$.

Given $a$ in $\mathbb{B}_{n}$, we denote by $g_{\alpha, a}$ the following function $\mathbb{B}_{n} \rightarrow \mathbb{C}$ :

$$
g_{\alpha, a}(z):=\frac{\left(1-|a|^{2}\right)^{\frac{n+1+\alpha}{2}}}{(1-\langle z, a\rangle)^{n+1+\alpha}} .
$$

This function and their properties stated below appear in Vukotić [28]. See also [29, Proposition 1.13] or [10, formula (2.4)]. By (5.4),

$$
g_{\alpha, a}\left(\varphi_{a}(z)\right) g_{\alpha, a}(z)=1 \text {. }
$$

By (5.9), (5.3), and (5.5),

$$
\left|g_{\alpha, a}\left(\varphi_{a}(w)\right)\right|^{2}\left(J_{\mathbb{R}} \varphi_{a}\right)(w)\left(1-\left|\varphi_{a}(w)\right|^{2}\right)^{\alpha}=\left(1-|w|^{2}\right)^{\alpha} .
$$

Using (5.10) and the change of variables $w=\varphi_{a}(z)$, one easily shows that for every $f$ in $f \in L^{2}\left(\mathbb{B}_{n}, \mu_{\alpha}\right)$,

$$
\left\|\left(f \circ \varphi_{a}\right) \cdot g_{\alpha, a}\right\|_{L^{2}\left(\mathbb{B}_{n}, \mu_{\alpha}\right)}=\|f\|_{L^{2}\left(\mathbb{B}_{n}, \mu_{\alpha}\right)} .
$$

\section{A weighted shift operator preserving $\mathcal{A}_{m}^{2}\left(\mathbb{B}_{n}, \mu_{\alpha}\right)$}

Definition 5.5. Given $a$ in $\mathbb{B}_{n}$, we define $U_{a}: \mathcal{A}_{m}^{2}\left(\mathbb{B}_{n}, \mu_{\alpha}\right) \rightarrow \mathcal{A}_{m}^{2}\left(\mathbb{B}_{n}, \mu_{\alpha}\right)$ by

$$
\left(U_{a} f\right)(z):=f\left(\varphi_{a}(z)\right) p_{m, a}(z) g_{\alpha, a}(z) .
$$


Proposition 5.6. Let $a \in \mathbb{B}^{n}$. Then $U_{a}$ is a unitary operator in $\mathcal{A}_{m}^{2}\left(\mathbb{B}_{n}, \mu_{\alpha}\right)$, and $U_{a}^{2}=I$.

Proof. Given $f$ in $\mathcal{A}_{m}^{2}\left(\mathbb{B}_{n}, \mu_{\alpha}\right)$, Lemma 5.3 assures that $U_{a} f \in \mathcal{A}_{m}\left(\mathbb{B}_{n}\right)$. Formula (5.11), combined with (5.6), implies that $U_{a}$ is an isometry. Finally, (5.7) and (5.9) yield the involutive property $U_{a}^{2}=I$.

\section{Computation of the RK on the unit ball}

Recall that $R_{m}^{(\alpha, \beta)}$ is defined by (3.5) and $\rho_{\mathbb{B}_{n}}(z, w)$ denotes $\left|\varphi_{z}(w)\right|$.

Theorem 5.7. Let $n, m \in \mathbb{N}$ and $\alpha>-1$. Then for every $z$ in $\mathbb{B}_{n}$, the following function $K_{z}$ is the reproducing kernel of $\mathcal{A}_{m}^{2}\left(\mathbb{B}_{n}, \mu_{\alpha}\right)$ at the point $z$ :

$$
K_{z}(w)=\frac{(1-\langle z, w\rangle)^{m-1}}{(1-\langle w, z\rangle)^{n+m+\alpha}} R_{m-1}^{(\alpha, n-1)}\left(\rho_{\mathbb{B}_{n}}(z, w)^{2}\right) .
$$

Proof. For $z=0$, the function defined by the right-hand side of (5.12) simplifies to

$$
K_{0}(w)=R_{m-1}^{(\alpha, n-1)}\left(|w|^{2}\right) .
$$

Theorem 3.3 means that $K_{0}$ is indeed the RK at the point 0 . Now, for $z$ in $\mathbb{B}_{n}$, we apply Proposition 4.2 with $H_{1}=H_{2}=\mathcal{A}_{m}^{2}\left(\mathbb{B}_{n}\right), \varphi_{z}$ instead of $\psi$, and $J_{z}:=p_{m, z} g_{\alpha, z}$. Since $\varphi_{z}(z)=0$, we obtain

$$
K_{z}(w)=\overline{J_{z}(z)} J_{z}(w) K_{0}\left(\varphi_{z}(w)\right) .
$$

It is easy to see that $J_{z}(z)=\left(1-|z|^{2}\right)^{-\frac{n+1+\alpha}{2}}$. So, after some simplifications, we arrive at (5.12):

$$
\begin{aligned}
K_{z}(w) & =\frac{1}{\left(1-|z|^{2}\right)^{\frac{n+1+\alpha}{2}}}\left(\frac{1-\langle z, w\rangle}{1-\langle w, z\rangle}\right)^{m-1} \frac{\left(1-|z|^{2}\right)^{\frac{n+1+\alpha}{2}}}{(1-\langle w, z\rangle)^{n+1+\alpha}} R_{m-1}^{(\alpha, n-1)}\left(\left|\varphi_{z}(w)\right|^{2}\right) \\
& =\frac{(1-\langle z, w\rangle)^{m-1}}{(1-\langle w, z\rangle)^{n+m+\alpha}} R_{m-1}^{(\alpha, n-1)}\left(\rho_{\mathbb{B}_{n}}(z, w)^{2}\right) .
\end{aligned}
$$

Formula (5.12) is a natural generalization of previous results: $[16,19]$ for $n=1$ and $\alpha=0$, [13] for $n=1$ and $\alpha>-1$, and [29, Theorem 2.7] for $m=1$.

Corollary 5.8. Let $n, m \in \mathbb{N}$ and $\alpha>-1$. Then for every $z$ in $\mathbb{B}_{n}$,

$$
\left\|K_{z}\right\|_{\mathcal{A}_{m}^{2}\left(\mathbb{B}_{n}, \mu_{\alpha}\right)}^{2}=K_{z}(z)=\left(\begin{array}{c}
n+m-1 \\
n
\end{array}\right) \frac{\mathrm{B}(\alpha+1, n)}{\mathrm{B}(\alpha+m, n)} \frac{1}{\left(1-|z|^{2}\right)^{n+\alpha+1}} .
$$


Remark 5.9. We get other formulas, equivalent to (5.12), using (5.3) and (3.2):

$$
\begin{aligned}
K_{z}(w)= & \frac{(1-\langle z, w\rangle)^{m-1}}{(1-\langle w, z\rangle)^{n+m+\alpha}} \frac{(-1)^{m-1} \mathrm{~B}(\alpha+1, n)}{\mathrm{B}(\alpha+m, n)} P_{m-1}^{(\alpha, n)}\left(2 \rho_{\mathbb{B}_{n}}(z, w)^{2}-1\right) \\
= & \frac{(1-\langle z, w\rangle)^{m-1}}{(1-\langle w, z\rangle)^{n+m+\alpha}} \frac{(-1)^{m-1} \mathrm{~B}(\alpha+1, n)}{\mathrm{B}(\alpha+m, n)} P_{m-1}^{(\alpha, n)}\left(1-\frac{2\left(1-|z|^{2}\right)\left(1-|w|^{2}\right)}{|1-\langle w, z\rangle|^{2}}\right) \\
= & \frac{(1-\langle z, w\rangle)^{m-1}}{(1-\langle w, z\rangle)^{n+m+\alpha}} \frac{(-1)^{m-1} \Gamma(\alpha+1)}{\Gamma(\alpha+n+1)(m-1) !} \times \\
& \quad \times \sum_{s=0}^{m-1}(-1)^{s}\left(\begin{array}{c}
m-1 \\
s
\end{array}\right) \frac{\Gamma(\alpha+m+n+s)}{\Gamma(\alpha+s+1)}\left(\frac{\left(1-|z|^{2}\right)\left(1-|w|^{2}\right)}{|1-\langle w, z\rangle|^{2}}\right)^{s}
\end{aligned}
$$

Remark 5.10. If $M$ is a unitary $n$ by $n$ matrix, then the RK computed in Theorem 5.7 is invariant under the simultaneous action of $M$ in both arguments:

$$
K_{M z}(M w)=K_{z}(w) \quad\left(z, w \in \mathbb{B}^{n}\right) .
$$

Therefore, by [18, Proposition 4.1], the space $\mathcal{A}_{m}^{2}\left(\mathbb{B}^{n}, \mu_{\alpha}\right)$ is invariant under the action of the rotation operator

$$
\left(R_{M} f\right)(z):=f\left(M^{-1} z\right) .
$$

This follows also directly from Proposition 2.7. Notice that the unitary matrices include permutation matrices, diagonal matrices with unimodular complex entries, and real rotations in any two coordinates.

Remark 5.11. Generalizing ideas of this section, it is possible to construct a unitary weighted shift operator $U_{\varphi}$ acting in $\mathcal{A}_{m}^{2}\left(\mathbb{B}_{n}, \mu_{\alpha}\right)$, for every biholomorphism $\varphi$ of $\mathbb{B}_{n}$.

The next result was published by Engliš [10, Section 2] for RKHS of harmonic functions. We reformulate it for our situation and recall the idea of the proof.

Proposition 5.12. Let $H=\mathcal{A}_{m}^{2}\left(\mathbb{B}_{n}, \mu_{\alpha}\right)$, with $n \geq 1$ and $m \geq 2$. Then $\operatorname{Ber}_{H}$ is not injective.

Proof. The functions $f(z)=z_{1}$ and $g(z)=\overline{z_{1}}$ are linearly independent elements of $H$. Therefore, the operator $S h=\langle h, f\rangle_{H} f-\langle h, g\rangle_{H} g$ is not zero, but the Berezin transform maps it into the zero function. 


\section{Reproducing kernel on the Siegel domain}

Let $n, m \in \mathbb{N}$ and $\alpha>-1$. In this section we compute the RK of the space $\mathcal{A}_{m}^{2}\left(\mathbb{H}_{n}, \nu_{\alpha}\right)$, where $\mathbb{H}_{n}$ is the standard Siegel domain (which can be considered as an unbounded realization of the unit ball) and $\nu_{\alpha}$ is a usual weighted measure on $\mathbb{H}_{n}$ :

$$
\begin{gathered}
\mathbb{H}_{n}:=\left\{\xi=\left(\xi^{\prime}, \xi_{n}\right) \in \mathbb{C}^{n-1} \times \mathbb{C}: \operatorname{Im}\left(\xi_{n}\right)-\left|\xi^{\prime}\right|^{2}>0\right\} \\
\mathrm{d} \nu_{\alpha}(\xi):=\frac{c_{\alpha}}{4}\left(\operatorname{Im}\left(\xi_{n}\right)-\left|\xi^{\prime}\right|^{2}\right)^{\alpha} \mathrm{d} \mu(\xi)
\end{gathered}
$$

For this purpose, we will construct a unitary operator $V: \mathcal{A}_{m}^{2}\left(\mathbb{B}_{n}, \mu_{\alpha}\right) \rightarrow \mathcal{A}_{m}^{2}\left(\mathbb{H}_{n}, \nu_{\alpha}\right)$, using some recipes from [21, Section 2] and an analog of the Pessoa factor which helps to preserve the polyanalyticity.

\section{Cayley transform}

Following [21, Section 2], we employ the biholomorphism $\omega: \mathbb{B}_{n} \rightarrow \mathbb{H}_{n}$ defined by

$$
\omega(z):=\left(\mathrm{i} \frac{z_{1}}{1+z_{n}}, \ldots, \mathrm{i} \frac{z_{n-1}}{1+z_{n}}, \mathrm{i} \frac{1-z_{n}}{1+z_{n}}\right) .
$$

Its inverse $\psi: \mathbb{H}_{n} \rightarrow \mathbb{B}_{n}$ is given by

$$
\psi(\xi):=\left(-\frac{2 \mathrm{i} \xi_{1}}{1-\mathrm{i} \xi_{n}}, \ldots,-\frac{2 \mathrm{i} \xi_{n-1}}{1-\mathrm{i} \xi_{n}}, \frac{1+\mathrm{i} \xi_{n}}{1-\mathrm{i} \xi_{n}}\right) .
$$

By a direct computation,

$$
1-\langle\psi(\xi), \psi(\eta)\rangle=4 \frac{\frac{\xi_{n}-\overline{\eta_{n}}}{2 \mathrm{i}}-\left\langle\xi^{\prime}, \eta^{\prime}\right\rangle}{\left(1-i \xi_{n}\right)\left(1+i \overline{\eta_{n}}\right)} .
$$

In particular,

$$
1-|\psi(\xi)|^{2}=4 \frac{\operatorname{Im}\left(\xi_{n}\right)-\left|\xi^{\prime}\right|^{2}}{\left|1-i \xi_{n}\right|^{2}} .
$$

The complex Jacobian matrices of $\psi$ and $\omega$ are triangular, and their determinants are easy to compute:

$$
\left(J_{\mathbb{C}} \omega\right)(z)=-\frac{2 \mathrm{i}^{n}}{\left(1+z_{n}\right)^{n+1}}, \quad\left(J_{\mathbb{C}} \psi\right)(\xi)=-\frac{(-2 \mathrm{i})^{n}}{\left(1-\mathrm{i} \xi_{n}\right)^{n+1}} .
$$

Therefore, the real Jacobians of $\omega$ and $\psi$ are

$$
\left(J_{\mathbb{R}} \omega\right)(z)=\frac{4}{\left|1+z_{n}\right|^{2(n+1)}}, \quad\left(J_{\mathbb{R}} \psi\right)(\xi)=\frac{4^{n}}{\left|1-\mathrm{i} \xi_{n}\right|^{2(n+1)}} .
$$




\section{Pseudohyperbolic distance on the Siegel domain}

Definition 6.1. Define a distance on $\mathbb{H}_{n}$ by

$$
\rho_{\mathbb{H}_{n}}(\xi, \eta):=\rho_{\mathbb{B}_{n}}(\psi(\xi), \psi(\eta)) .
$$

The following proposition provides an efficient formula to compute $\rho_{\mathbb{H}_{n}}(\xi, \eta)$.

Proposition 6.2. For every $\xi, \eta$ in $\mathbb{H}_{n}$,

$$
1-\rho_{\mathbb{H}_{n}}(\xi, \eta)^{2}=\frac{\left(\operatorname{Im}\left(\xi_{n}\right)-\left|\xi^{\prime}\right|^{2}\right)\left(\operatorname{Im}\left(\eta_{n}\right)-\left|\eta^{\prime}\right|^{2}\right)}{\left|\frac{\xi_{n}-\overline{\eta_{n}}}{2 \mathrm{i}}-\left\langle\xi^{\prime}, \eta^{\prime}\right\rangle\right|^{2}} .
$$

Proof. Substitute $\psi(\xi)$ and $\psi(\eta)$ instead of $z$ and $w$ in (5.3):

$$
1-\rho_{\mathbb{H}_{n}}(\xi, \eta)^{2}=1-\rho_{\mathbb{B}_{n}}(\psi(\xi), \psi(\eta))^{2}=\frac{\left(1-|\psi(\xi)|^{2}\right)\left(1-|\psi(\eta)|^{2}\right)}{|1-\langle\psi(\xi), \psi(\eta)\rangle|^{2}} .
$$

Applying (6.3) and (6.4) we obtain (6.8).

Remark 6.3. For $n=1$, formulas (6.7) and (6.8) simplify to

$$
\rho_{\mathbb{H}_{1}}(\xi, \eta)=\frac{|\xi-\eta|}{|\bar{\xi}-\eta|}, \quad 1-\rho_{\mathbb{H}_{1}}(\xi, \eta)^{2}=\frac{4 \operatorname{Im}(\xi) \operatorname{Im}(\eta)}{|\bar{\xi}-\eta|^{2}} .
$$

\section{A factor to preserve the norm when passing from $\mathbb{H}_{n}$ to $\mathbb{B}_{n}$}

The material of this subsection is equivalent to some computations from [21, Section 2]. Define $h_{\alpha}: \mathbb{H}_{n} \rightarrow \mathbb{C}$ by

$$
h_{\alpha}(\xi):=\left(\frac{2}{1-\mathrm{i} \xi_{n}}\right)^{n+\alpha+1}
$$

Lemma 6.4. For every $\xi$ in $\mathbb{H}_{n}$,

$$
\left|h_{\alpha}(\xi)\right|^{2}=\frac{4\left(1-|\psi(\xi)|^{2}\right)^{\alpha}\left(J_{\mathbb{R}} \psi\right)(\xi)}{\left(\operatorname{Im}\left(\xi_{n}\right)-\left|\xi^{\prime}\right|^{2}\right)^{\alpha}} .
$$

For any $z$ in $\mathbb{B}_{n}$

$$
\frac{1}{4}\left|h_{\alpha}(\omega(z))\right|^{2}\left(\frac{1-|z|^{2}}{\left|1+z_{n}\right|^{2}}\right)^{\alpha}\left(J_{\mathbb{R}} \omega\right)(z)=\left(1-|z|^{2}\right)^{\alpha} .
$$

Proof. Formula (6.11) is obtained by (6.4) and (6.6). Then (6.12) follows from (6.11) and the well-known formula for the Jacobian of the inverse function. 
Lemma 6.5. Let $u \in L^{2}\left(\mathbb{B}_{n}, \mu_{\alpha}\right)$. Then

$$
\left\|(u \circ \psi) \cdot h_{\alpha}\right\|_{L^{2}\left(\mathbb{H}_{n}, \nu_{\alpha}\right)}=\|u\|_{L^{2}\left(\mathbb{B}_{n}, \mu_{\alpha}\right)} .
$$

Proof. First, using (6.4), we observe that the change of variable $z=\psi(\zeta)$ transforms the weight function in the following way:

$$
\left(\operatorname{Im}\left(\zeta_{n}\right)-\left|\zeta^{\prime}\right|^{2}\right)^{\alpha}=\left(\frac{1-|z|^{2}}{\left|1+z_{n}\right|^{2}}\right)^{\alpha} .
$$

Apply this change of variables in the integral:

$$
\begin{aligned}
\left\|(u \circ \psi) \cdot h_{\alpha}\right\|_{L^{2}\left(\mathbb{H}_{n}, \nu_{\alpha}\right)}^{2} & =\frac{c_{\alpha}}{4} \int_{\mathbb{H}^{n}}|u(\psi(\zeta))|^{2}\left|h_{\alpha}(\zeta)\right|^{2}\left(\operatorname{Im}\left(\zeta_{n}\right)-\left|\zeta^{\prime}\right|^{2}\right)^{\alpha} \mathrm{d} \mu(\zeta) \\
& =c_{\alpha} \int_{\mathbb{B}^{n}}|u(z)|^{2}\left|h_{\alpha}(\omega(z))\right|^{2}\left(\frac{1-|z|^{2}}{\left|1+z_{n}\right|^{2}}\right)^{\alpha}\left(J_{\mathbb{R}} \omega\right)(z) \mathrm{d} \mu(z) \\
& =c_{\alpha} \int_{\mathbb{B}^{n}}|u(z)|^{2}\left(1-|z|^{2}\right)^{\alpha} \mathrm{d} \mu(z)=\|u\|_{L^{2}\left(\mathbb{B}_{n}, \mu_{\alpha}\right)}^{2}
\end{aligned}
$$

\section{A factor to preserve the polyanalyticity when passing from the unit ball to the Siegel domain}

Definition 6.6. Define $q_{m}: \mathbb{H}_{n} \rightarrow \mathbb{C}$,

$$
q_{m}(\xi):=\left(\frac{1+\mathrm{i} \overline{\xi_{n}}}{1-\mathrm{i} \xi_{n}}\right)^{m-1} .
$$

Lemma 6.7. Let $f \in \mathcal{A}_{m}\left(\mathbb{B}_{n}\right)$. Then $(f \circ \psi) \cdot q_{m} \in \mathcal{A}_{m}\left(\mathbb{H}_{n}\right)$.

Proof. This proof is similar to the proof of Lemma 5.3. The main idea is that the factor $\left(1+\mathrm{i} \overline{\xi_{n}}\right)^{m-1}$, appearing in the numerator of $q_{m}(\xi)$, cancels the denominators of the expressions $\overline{\psi(\xi)}^{j}$, where $|j|<m$. We represent $f$ in the form (1.4), compose with $\psi$, and multiply by $q_{m}$ :

$$
\begin{aligned}
u(\xi) & :=(f \circ \psi)(\xi) q_{m}(\xi)=\sum_{|j| \leq m} h_{j}(\psi(\xi))\left(\prod_{s=1}^{n-1} \frac{\left(2 \mathrm{i} \overline{\xi_{s}}\right)^{j_{s}}}{\left(1+\mathrm{i} \overline{\xi_{n}}\right)^{j_{s}}}\right) \frac{\left(1-\mathrm{i} \overline{\xi_{n}}\right)^{j_{n}}}{\left(1+\mathrm{i} \overline{\xi_{n}}\right)^{j_{n}}} \frac{\left(1+\mathrm{i} \overline{\xi_{n}}\right)^{m-1}}{\left(1-\mathrm{i} \xi_{n}\right)^{m-1}} \\
& =\sum_{|j| \leq m} \frac{h_{j}(\psi(\xi))}{\left(1-\mathrm{i} \xi_{n}\right)^{m-1}}\left(\prod_{s=1}^{n-1}\left(2 \mathrm{i} \overline{\xi_{s}}\right)^{j_{s}}\right)\left(1-\mathrm{i} \overline{\xi_{n}}\right)^{j_{n}}\left(1+\mathrm{i} \overline{\xi_{n}}\right)^{m-|j|-1} .
\end{aligned}
$$

For each $j$, the corresponding summand is the product of an analytic function by a polynomial in $\overline{\xi_{1}}, \ldots, \overline{\xi_{n}}$ of total degree $m-1$. 
Remark 6.8. Another way to prove Lemma 6.7, computing $\bar{D}^{j} u$, seems to be more complicated. We will show it only for $n=2$ and $m=2$. In this case,

$$
u(\xi)=\frac{1+\mathrm{i} \overline{\xi_{2}}}{1-\mathrm{i} \xi_{2}} f\left(-\frac{2 \mathrm{i} \xi_{1}}{1-\mathrm{i} \xi_{2}}, \frac{1+\mathrm{i} \xi_{2}}{1-\mathrm{i} \xi_{2}}\right),
$$

By the well-known chain rule and product rule for Wirtinger derivatives,

$$
\begin{aligned}
\left(\bar{D}^{(1,0)} u\right)(\xi) & =\frac{2 \mathrm{i}\left(\bar{D}^{(1,0)} f\right)(\psi(\xi))}{1-\mathrm{i} \xi_{2}}, \\
\left(\bar{D}^{(0,1)} u\right)(\xi) & =\frac{\mathrm{i} f(\psi(\xi))}{1-\mathrm{i} \xi_{2}}+\frac{2\left(\left(\overline{\xi_{1}} \bar{D}^{(1,0)}-\mathrm{i} \bar{D}^{(0,1)}\right) f\right)(\psi(\xi))}{\left(1-\mathrm{i} \xi_{2}\right)\left(1+\mathrm{i} \overline{\xi_{2}}\right)} \\
\left(\bar{D}^{(2,0)} u\right)(\xi) & =\frac{-4\left(\bar{D}^{(2,0)} f\right)(\psi(\xi))}{\left(1-\mathrm{i} \xi_{2}\right)\left(1+\mathrm{i} \overline{\xi_{2}}\right)}, \\
\left(\bar{D}^{(1,1)} u\right)(\xi) & =\frac{4\left(\left(\overline{\mathrm{i}}_{1} \bar{D}^{(2,0)}+\bar{D}^{(1,1)}\right) f\right)(\psi(\xi))}{\left(1-\mathrm{i} \xi_{2}\right)\left(1+\mathrm{i} \overline{\xi_{2}}\right)^{2}}, \\
\left(\bar{D}^{(0,2)} u\right)(\xi) & =\frac{4\left(\left(\bar{\xi}_{2}^{2} \bar{D}^{(2,0)}-2 \mathrm{i} \bar{\xi}_{1} \bar{D}^{(1,1)}-\bar{D}^{(0,2)}\right) f\right)(\psi(\xi))}{\left(1-\mathrm{i} \xi_{2}\right)\left(1+\mathrm{i} \bar{\xi}_{2}\right)^{3}} .
\end{aligned}
$$

Since $f \in \mathcal{A}_{2}\left(\mathbb{B}_{n}\right)$, we conclude that $u \in \mathcal{A}_{2}\left(\mathbb{H}_{n}\right)$.

\section{A weighted change of variables which unitarily maps $\mathcal{A}_{m}^{2}\left(\mathbb{B}_{n}, \mu_{\alpha}\right)$ onto $\mathcal{A}_{m}^{2}\left(\mathbb{H}_{n}, \nu_{\alpha}\right)$}

Definition 6.9. Define $V: \mathcal{A}_{m}^{2}\left(\mathbb{B}_{n}, \mu_{\alpha}\right) \rightarrow \mathcal{A}_{m}^{2}\left(\mathbb{H}_{n}, \nu_{\alpha}\right)$ by $V u:=(u \circ \psi) \cdot h_{\alpha} \cdot q_{m}$, i.e.,

$$
(V u)(\xi):=u(\psi(\xi)) h_{\alpha}(\xi) q_{m}(\xi) .
$$

Proposition 6.10. $V$ is a well-defined unitary operator $\mathcal{A}_{m}^{2}\left(\mathbb{B}_{n}, \mu_{\alpha}\right) \rightarrow \mathcal{A}_{m}^{2}\left(\mathbb{H}_{n}, \nu_{\alpha}\right)$.

Proof. Lemma 6.7 assures that $V u \in \mathcal{A}_{m}\left(\mathbb{H}_{n}\right)$ for every $u$ in $\mathcal{A}_{m}^{2}\left(\mathbb{B}_{n}, \mu_{\alpha}\right)$. Lemma 6.5, combined with the identity $\left|q_{m}(\xi)\right|=1$, provides the isometric property of $V$. It is easy to verify that the adjoint operator $V^{*}$ acts by

$$
\left(V^{*} f\right)(z)=\frac{f(\omega(z))}{h_{\alpha}(\omega(z)) q_{m}(\omega(z))},
$$

and that $V^{*}$ is the inverse operator to $V$. 


\section{Computation of the RK on the Siegel domain}

We define $t^{\beta}$ via the principal argument of $t$, see Remark 5.4. The formulas $(t u)^{\beta}=t^{\beta} u^{\beta}$ and $(t / u)^{\beta}$ are not always true. Let us recall some sufficient conditions for these formulas to be true.

Lemma 6.11. Let $t, u \in \mathbb{C} \backslash\{0\}$ and $\beta \in \mathbb{C}$.

1. If $\operatorname{Re}(t)>0$ and $\operatorname{Re}(u)>0$, then $(t u)^{\beta}=t^{\beta} u^{\beta}$.

2. If $\operatorname{Re}(t)>0$ and $\operatorname{Re}(t / u)>0$, then $(t / u)^{\beta}=t^{\beta} / u^{\beta}$.

Proof. 1. The assumptions on $t$ and $u$ imply that $\arg (t u)=\arg (t)+\arg (u)$.

2. Follows from part 1 applied to $t$ and $u / t$.

Lemma 6.12. Let $\xi, \eta \in \mathbb{H}_{n}$ and $\beta \geq 0$. Then

$$
(1-\langle\psi(\xi), \psi(\eta)\rangle)^{\beta}=\frac{4^{\beta}\left(\frac{\xi_{n}-\overline{\eta_{n}}}{2 \mathrm{i}}-\left\langle\xi^{\prime}, \eta^{\prime}\right\rangle\right)^{\beta}}{\left(1-\mathrm{i} \xi_{n}\right)^{\beta}\left(1+\mathrm{i} \overline{\eta_{n}}\right)^{\beta}} .
$$

Proof. Due to (6.3), $1-\langle\psi(\xi), \psi(\eta)\rangle=t /(u v)$, where

$$
t:=4\left(\frac{\xi_{n}-\overline{\eta_{n}}}{2 \mathrm{i}}-\left\langle\xi^{\prime}, \eta^{\prime}\right\rangle\right), \quad u:=1-\mathrm{i} \xi_{n}, \quad v:=1+\mathrm{i} \overline{\eta_{n}}
$$

Since $|\psi(\xi)|<1$ and $|\psi(\eta)|<1$, we obtain

$$
\operatorname{Re}(t /(u v))=\operatorname{Re}(1-\langle\psi(\xi), \psi(\eta)\rangle)>0 .
$$

Furthermore, $\operatorname{Re}(u)=1+\operatorname{Im}\left(\xi_{n}\right)>0$ and $\operatorname{Re}(v)=1+\operatorname{Im}\left(\eta_{n}\right)>0$. So, by Lemma 6.11 ,

$$
\left(\frac{t}{u v}\right)^{\beta}=\frac{t^{\beta}}{(u v)^{\beta}}=\frac{t^{\beta}}{u^{\beta} v^{\beta}} .
$$

Theorem 6.13. Let $n, m \in \mathbb{N}$ and $\alpha>-1$. Then for every $\xi$ in $\mathbb{H}_{n}$, the following function $\widetilde{K}_{\xi}$ is the reproducing kernel of $\mathcal{A}_{m}^{2}\left(\mathbb{H}_{n}, \nu_{\alpha}\right)$ at the point $\xi$ :

$$
\widetilde{K}_{\xi}(\eta)=\frac{\left(\frac{\xi_{n}-\overline{\eta_{n}}}{2 \mathrm{i}}-\left\langle\xi^{\prime}, \eta^{\prime}\right\rangle\right)^{m-1}}{\left(\frac{\eta_{n}-\overline{\xi_{n}}}{2 \mathrm{i}}-\left\langle\eta^{\prime}, \xi^{\prime}\right\rangle\right)^{n+m+\alpha}} R_{m-1}^{(\alpha, n-1)}\left(\rho_{\mathbb{H}_{n}}(\xi, \eta)^{2}\right) .
$$

Proof. Due to Proposition 6.10, we can apply Proposition 4.1 with $H_{1}=\mathcal{A}_{m}\left(\mathbb{B}_{n}, \mu_{\alpha}\right)$, $H_{2}=\mathcal{A}_{m}\left(\mathbb{H}_{n}, \nu_{\alpha}\right)$, and $J(\xi):=h_{\alpha}(\xi) q_{m}(\xi)$. So, for every $\xi$ in $\mathbb{H}_{n}$, the next function is the RK of $\mathcal{A}_{m}\left(\mathbb{H}_{n}, \nu_{\alpha}\right)$ associated to the point $\xi$ :

$$
\widetilde{K}_{\xi}(\eta)=\overline{h_{\alpha}(\xi) q_{m}(\xi)} h_{\alpha}(\eta) q_{m}(\eta) K_{\psi(\xi)}(\psi(\eta))
$$


Substitute formula (5.12) for $K$ :

$$
\widetilde{K}_{\xi}(\eta)=\overline{h_{\alpha}(\xi) q_{m}(\xi)} h_{\alpha}(\eta) q_{m}(\eta) \frac{(1-\langle\psi(\xi), \psi(\eta)\rangle)^{m-1}}{(1-\langle\psi(\eta), \psi(\xi)\rangle)^{n+m+\alpha}} R_{m-1}^{(\alpha, n-1)}\left(\rho_{\mathbb{H}_{n}}(\xi, \eta)^{2}\right) .
$$

Then, substitute the definitions of $h_{\alpha}, q_{m}$ and use Lemma 6.12:

$$
\begin{aligned}
\widetilde{K}_{\xi}(\eta)= & R_{m-1}^{(\alpha, n-1)}\left(\rho_{\mathbb{H}_{n}}(\xi, \eta)^{2}\right) \frac{2^{n+\alpha+1}\left(1+\mathrm{i} \overline{\eta_{n}}\right)^{m-1}}{\left(1-\mathrm{i} \eta_{n}\right)^{n+m+\alpha}} \frac{2^{n+\alpha+1}\left(1-\mathrm{i} \xi_{n}\right)^{m-1}}{\left(1+\mathrm{i} \overline{\xi_{n}}\right)^{n+m+\alpha}} \\
& \times \frac{4^{m-1}\left(\frac{\xi_{n}-\overline{\eta_{n}}}{2 \mathrm{i}}-\left\langle\xi^{\prime}, \eta^{\prime}\right\rangle\right)^{m-1}}{\left(1-\mathrm{i} \xi_{n}\right)^{m-1}\left(1+\mathrm{i} \overline{\eta_{n}}\right)^{m-1}} \frac{\left(1+\mathrm{i} \overline{\xi_{n}}\right)^{n+m+\alpha}\left(1-\mathrm{i} \eta_{n}\right)^{n+m+\alpha}}{4^{n+m+\alpha}\left(\frac{\eta_{n}-\overline{\xi_{n}}}{2 \mathrm{i}}-\left\langle\eta^{\prime}, \xi^{\prime}\right\rangle\right)^{n+m+\alpha}} .
\end{aligned}
$$

Simplifying this expression we obtain the right-hand side of (6.15).

Corollary 6.14. Let $n, m \in \mathbb{N}$ and $\alpha>-1$. Then for every $\xi$ in $\mathbb{H}_{n}$,

$$
\left\|\widetilde{K}_{\xi}\right\|_{\mathcal{A}_{m}^{2}\left(\mathbb{H}_{n}, \nu_{\alpha}\right)}^{2}=\widetilde{K}_{\xi}(\xi)=\left(\begin{array}{c}
n+m-1 \\
n
\end{array}\right) \frac{\mathrm{B}(\alpha+1, n)}{\mathrm{B}(\alpha+m, n)} \frac{1}{\left(\operatorname{Im}\left(\xi_{n}\right)-\left|\xi^{\prime}\right|^{2}\right)^{\alpha+n+1}} .
$$

Remark 6.15. Analogously to the case of the unit ball, we get some formulas equivalent to (6.15), using (6.8) and (3.2):

$$
\begin{aligned}
\widetilde{K}_{\xi}(\eta)= & \frac{\left(\frac{\xi_{n}-\overline{\eta_{n}}}{2 \mathrm{i}}-\left\langle\xi^{\prime}, \eta^{\prime}\right\rangle\right)^{m-1}}{\left(\frac{\eta_{n}-\overline{\bar{\xi}_{n}}}{2 \mathrm{i}}-\left\langle\eta^{\prime}, \xi^{\prime}\right\rangle\right)^{n+m+\alpha}} \frac{(-1)^{m-1} \mathrm{~B}(\alpha+1, n)}{\mathrm{B}(\alpha+m, n)} P_{m-1}^{(\alpha, n)}\left(2 \rho_{\mathbb{H}_{n}}(\xi, \eta)^{2}-1\right) \\
= & \frac{\left(\frac{\xi_{n}-\overline{\eta_{n}}}{2 \mathrm{i}}-\left\langle\xi^{\prime}, \eta^{\prime}\right\rangle\right)^{m-1}}{\left(\frac{\eta_{n} \overline{\xi_{n}}}{2 \mathrm{i}}-\left\langle\eta^{\prime}, \xi^{\prime}\right\rangle\right)^{n+m+\alpha}} \frac{(-1)^{m-1} \mathrm{~B}(\alpha+1, n)}{\mathrm{B}(\alpha+m, n)} \times \\
& \times P_{m-1}^{(\alpha, n)}\left(1-\frac{2\left(\operatorname{Im}\left(\xi_{n}\right)-\left|\xi^{\prime}\right|^{2}\right)\left(\operatorname{Im}\left(\eta_{n}\right)-\left|\eta^{\prime}\right|^{2}\right)}{\left|\frac{\xi_{n}-\overline{\eta_{n}}}{2 \mathrm{i}}-\left\langle\xi^{\prime}, \eta^{\prime}\right\rangle\right|^{2}}\right) \\
= & \frac{\left(\frac{\xi_{n}-\overline{\eta_{n}}}{2 \mathrm{i}}-\left\langle\xi^{\prime}, \eta^{\prime}\right\rangle\right)^{m-1}}{\left(\frac{\eta_{n} \overline{\xi_{n}}}{2 \mathrm{i}}-\left\langle\eta^{\prime}, \xi^{\prime}\right\rangle\right)^{n+m+\alpha}} \frac{(-1)^{m-1} \Gamma(\alpha+1)}{\Gamma(\alpha+n+1)(m-1) !} \times \\
& \quad \times \sum_{s=0}^{m-1}(-1)^{s}\left(\begin{array}{c}
m-1 \\
s
\end{array}\right) \frac{\Gamma(\alpha+m+n+s)}{\Gamma(\alpha+s+1)}\left(\frac{\left(\operatorname{Im}\left(\xi_{n}\right)-\left|\xi^{\prime}\right|^{2}\right)\left(\operatorname{Im}\left(\eta_{n}\right)-\left|\eta^{\prime}\right|^{2}\right)}{\left|\frac{\xi_{n}-\overline{\eta_{n}}}{2 \mathrm{i}}-\left\langle\xi^{\prime}, \eta^{\prime}\right\rangle\right|^{2}}\right)^{s} .
\end{aligned}
$$

Remark 6.16. In the case $n=1$, i.e., for the upper halfplane $\mathbb{H}_{1}$, formula (6.15) simplifies to

$$
\widetilde{K}_{\xi}(\eta)=\frac{\left(\frac{\xi-\bar{\eta}}{2 \mathrm{i}}\right)^{m-1}}{\left(\frac{\eta-\bar{\xi}}{2 \mathrm{i}}\right)^{m+\alpha+1}} R_{m-1}^{(\alpha, 0)}\left(\frac{|\xi-\eta|^{2}}{|\bar{\xi}-\eta|^{2}}\right) \quad\left(\xi, \eta \in \mathbb{H}_{1}\right) .
$$


In particular, for $\alpha=0$, this expression coincides with formula [19, Corollary 2.5] obtained by another method.

Remark 6.17. Generalizing ideas of this paper, it is possible to associate a unitary operator (namely, a certain weighted shift) in $\mathcal{A}_{m}^{2}\left(\mathbb{H}_{n}, \nu_{\alpha}\right)$ to every biholomorphism of the Siegel domain $\mathbb{H}_{n}$. In particular, using (6.15), we have verified that the space $\mathcal{A}_{m}\left(\mathbb{H}_{n}, \nu_{\alpha}\right)$ is invariant under the unweighted changes of variables, corresponding to the quasi-parabolic, nilpotent, and quasi-nilpotent groups from [21, Section 3].

\section{Acknowledgements}

The authors have been partially supported by Proyecto CONACYT "Ciencia de Frontera" FORDECYT-PRONACES/61517/2020, by CONACYT (Mexico) scholarships, and by SIP-IPN projects (Instituto Politécnico Nacional, Mexico). We are grateful to professor

Nikolai Vasilevski for inviting us to this area of mathematics and to professor Armando Sánchez-Nungaray for the term "homogeneously polyanalytic function".

\section{References}

[1] Abreu, L.D.: On the structure of Gabor and super Gabor spaces. Monatsh. Math. 161, 237-253 (2010), doi:10.1007/s00605-009-0177-0.

[2] Abreu, L.D., Feichtinger, H.G.: Function spaces of polyanalytic functions. In: Harmonic and Complex Analysis and its Applications, Birkhäuser, 1-38 (2014), doi:10.1007/978-3-319-01806-5_1.

[3] Aronszajn, N.: Theory of reproducing kernels. Transactions of the AMS 68, 337-404 (1950), doi:10.1090/S0002-9947-1950-0051437-7.

[4] Askour, N., Intissar, A., Mouayn, Z.: Explicit formulas for reproducing kernels of generalized Bargmann spaces. C.R. Acad. Sci. Paris, Ser. I 325, 707-712 (1997), doi : 10.1016/S0764-4442(97)80045-6.

[5] Balk, M.B.: Polyanalytic Functions. Akademie Verlag, Berlin (1991).

[6] Barrera-Castelán, R.M., Maximenko, E.A., Ramos-Vazquez, G.: Radial operators on polyanalytic weighted Bergman spaces. Preprint: arXiv:2009.14301v2.

[7] Bergman, S.: The kernel function and conformal mapping. American Mathematical Society, New York (1950). 
[8] Čučković, Ž., Le, T.: Toeplitz operators on Bergman spaces of polyanalytic functions. Bull. London Math. Soc., 44, 961—973 (2012), doi:10.1112/blms/bds024.

[9] Duren, P., Weir, R.: The pseudohyperbolic metric and Bergman spaces in the ball. Trans. Amer. Math. Soc. 359, 63-76 (2007), http://www.jstor.org/stable/20161567.

[10] Engliš, M.: Toeplitz operators and group representations. J. Fourier Anal. Appl., 13, 243-265 (2007), doi:10.1007/s00041-006-6009-x.

[11] Faraut, J., Koranyi, A.: Function spaces and reproducing kernels on bounded symmetric domains. J. Funct. Anal. 88, 64-89 (1990), doi : 10.1016/0022-1236(90)90119-6.

[12] Fedorovsky, Yu.: Approximation by polyanalytic polynomials (in Russian). Keldysh Institute of Applied Math., Moscow (2016), doi:10.20948/mono-2016-fedorovsky.

[13] Hachadi, H., Youssfi, E.H.: The polyanalytic reproducing kernels. Complex Anal. Oper. Theory, 13, 3457-3478 (2019), doi:10.1007/s11785-019-00956-5.

[14] Haimi, A., Hedenmalm, H.: The polyanalytic Ginibre ensembles. J. Stat. Phys. 153, 10-47 (2013), doi : 10.1007/s10955-013-0813-x.

[15] Hutník, O., Hutníková, M.: Toeplitz operators on poly-analytic spaces via time-scale analysis. Oper. Matrices 8, 1107-1129 (2015), doi:10.7153/oam-08-62.

[16] Koshelev, A.D.: On the kernel function of the Hilbert space of functions polyanalytic in a disc. Dokl. Akad. Nauk SSSR 232, 277-279 (1977). English translation: Soviet Math. Dokl. 18(1), 59-62 (1977), http://mi.mathnet.ru/eng/dan40862.

[17] Loaiza M., Ramírez-Ortega, J.: Toeplitz operators with homogeneous symbols acting on the poly-Bergman spaces of the upper half-plane. Integral Equ. Oper. Theory 87, 391-410 (2017), doi:10.1007/s00020-017-2350-5.

[18] Maximenko, E.A., Tellería-Romero, A.M.: Radial operators in polyanalytic Bargmann-Segal-Fock spaces. Operator Theory: Adv. and Appl., Birkhäuser, 279, 277-305 (2020), doi : 10.1007/978-3-030-44651-2_18.

[19] Pessoa, L.V.: The method of variation of the domain for poly-Bergman spaces. Math. Nachr. 286, 1850-1862 (2013), doi :10.1002/mana.201010057.

[20] Pessoa, L.V.: Planar Beurling transform and Bergman type spaces. Complex Anal. Oper. Theory 8, 359-381 (2014), doi:10.1007/s11785-012-0268-0. 
[21] Quiroga-Barranco, R., Vasilevski, N.L.: Commutative C*-algebras of Toeplitz operators on the unit ball, I. Bargmann type transforms and spectral representations of Toeplitz operators. Integral Equ. Oper. Theory 59, 379-419 (2007), doi:10.1007/s00020-007-1537-6.

[22] Ramazanov, A.K.: Representation of the space of polyanalytic functions as a direct sum of orthogonal subspaces. Application to rational approximations, Math. Notes 66, 613-627 (1999), doi:10.1007/BF02674203.

[23] Ramírez Ortega, J., Sánchez Nungaray, A.: Poly-Bergman type spaces on the Siegel domain. Commun. Math. Anal. 14(2), 113-128 (2013), https://projecteuclid.org/euclid.cma/1356039036.

[24] Ramírez Ortega, J., Sánchez-Nungaray, A.: Toeplitz operators with vertical symbols acting on the poly-Bergman spaces of the upper half-plane. Complex Anal. Oper. Theory 9, 1801-1817 (2015), doi : 10.1007/s11785-015-0469-4.

[25] Rudin, W.: Function Theory on the Unit Ball of $\mathbb{C}^{n}$. Springer-Verlag, Berlin Heidelberg (1980), doi:10.1007/978-3-540-68276-9.

[26] Vasilevski, N.L.: Poly-Fock spaces. Operator Theory: Adv. and Appl. 117, Birkhäuser, 371-386 (2000), doi:10.1007/978-3-0348-8403-7_28.

[27] Vasilevski, N.L.: Commutative Algebras of Toeplitz Operators on the Bergman Space. Birkhäuser, Basel-Boston-Berlin (2008), doi :10.1007/978-3-7643-8726-6.

[28] Vukotić, D.: A sharp estimate for $A_{\alpha}^{p}$ functions in $\mathbb{C}^{n}$. Proc. Am. Math. Soc. 117, 753-756 (1993), doi:10.2307/2159138.

[29] Zhu, K.: Spaces of Holomorphic Functions in the Unit Ball. Springer-Verlag, New York (2005).

Christian Rene Leal-Pacheco

Centro de Investigación y de Estudios Avanzados del Instituto Politécnico Nacional

Departamento de Matemáticas

Apartado Postal 07360

Ciudad de México

Mexico

e-mail: christian.leal.pacheco@gmail.com

https://orcid.org/0000-0001-5738-4904

Egor A. Maximenko

Instituto Politécnico Nacional

Escuela Superior de Física y Matemáticas 
Apartado Postal 07730

Ciudad de México

Mexico

e-mail: egormaximenko@gmail.com

https://orcid.org/0000-0002-1497-4338

Gerardo Ramos-Vazquez

Centro de Investigación y de Estudios Avanzados del Instituto Politécnico Nacional Departamento de Matemáticas

Apartado Postal 07360

Ciudad de México

Mexico

e-mail: ger.ramosv@gmail.com

https://orcid.org/0000-0001-9363-8043 